\section{FDA approves first RET inhibitor}

The FDA has approved Eli Lilly's RET inhibitor Retevmo (selpercatinib) for three types of RET-driven cancer. The company acquired the first-in-class agent last year, in an $\$ 8$ billion acquisition of Loxo Oncology that broadened Lilly's oncology focus.

Around $2 \%$ of cancers carry mutations or abnormalities in the receptor tyrosine kinase RET. In the phase $1 / 2$ LIBRETTO-001 trial of Retevmo, the sponsors enrolled patients with advanced, RET-aberrant solid cancers. The data from this trial have now supported the FDA's approvals for Retevmo in non-small-cell lung cancer (NSCLC), one of the most prevalent forms of cancer, and in two forms of thyroid cancer, in which RET alterations are particularly common. The overall response rates to treatment were $64-85 \%$ in patients with RET-fusion-positive NSCLC, 69-73\% in patients with $R E T$-mutant medullary thyroid cancer, and $75-79 \%$ in patients with RET-fusion-positive thyroid cancer.

Lilly has not developed a companion diagnostic for Retevmo, and recommends next-generation sequencing or other approaches to identify patients who are most likely to respond to treatment. A tissue-agnostic approval - in which the drug is available for all patients with $R E T$ alterations, regardless of where the cancer started - could still be on the horizon for Retevmo. "Regulatory interactions are planned to understand what the registration path would be for a tissue-agnostic indication," a Lilly spokesperson told Nature Biotechnology. The LIBRETTO-001 trial is still enrolling patients with RET-driven tumors beyond the lung and thyroid, she added. Loxo and partner Bayer secured a landmark tissue-agnostic approval in 2019 for the NTRK inhibitor Vitrakvi (larotrectinib).

Others are also looking to compete in the RET inhibitor space. Blueprint Medicines submitted its pralsetinib for $R E T$-fusion-positive NSCLC earlier this year, paving the way for a possible approval by year end. Turning Point Therapeutics' dual RET and SRC inhibitor TPX-0046 is in phase $1 / 2$ testing.

Published online: 9 June 2020

https://doi.org/10.1038/s41587-020-0568-2 and Medical Devices for a phase 2 trial in hospitalized patients with COVID-19.

Another target class highlighted

by Krogan and his collaborators is stress-granule-related factors G3BP1 and G3BP2, captured by the virus's nucleocapsid protein. Stress granules come together in the cytoplasm by liquid-liquid phase separation, sequestering hundreds of RNAs and proteins and preventing translation initiation; they are often induced in response to viral infection. Some viruses are known to disrupt antiviral stress granules (which are enriched for G3BP1), but the precise mechanism by which they accomplish this remains unknown, says Craig McCormick at the Department of Microbiology \& Immunology, Dalhousie University, Canada. He suggests "as well as limiting viral replication, stress-granule-inducing drugs could facilitate the establishment of an antiviral state in uninfected cells."

In this respect, zotatifin and Aplidin may inhibit the translation of viral RNA by promoting stress-granule aggregation. Conversely, two drugs that prevent stress-granule disassembly could also prove useful. New Taipei City-based Senhwa Biosciences' silmitasertib, an experimental small-molecule inhibitor of the protein kinase CK2 in phase 2 testing for cancer, is now undergoing human testing against COVID-19 after the company signed an agreement with the US National Institute of Allergy and Infectious Diseases. Elsewhere, a non-peer-reviewed preprint on measured protein expression changes in Caco-2 cells during infection with SARS-CoV-2 identified Nerviano Medical Sciences' experimental drug NMS-873 as an inhibitor of stress-granule disassembly (among other things). The drug, which inhibits the AAA ATPase (an extended family of ATPases associated with various cellular activities) p97, inhibits viral replication at low nanomolar concentrations.

Not all of the 69 drugs identified in the Krogan paper are clear-cut home runs; for example, SARS-CoV-2 non-structural protein 5 (Nsp5; 3CLpro; Mpro) interacts with the epigenetic eraser histone deacetylase 2 (HDAC-2). The paper identifies the generic HDAC-2 inhibitor valproic acid (approved for bipolar disorder, migraine and epilepsy) as a possible drug candidate. But HDAC-2 transcriptional regulation is known to suppress proinflammatory responses from monocytes and macrophages in the lungs, so it seems more likely that an HDAC-2 agonist rather than inhibitor would be beneficial in the context of dampening ARDS in COVID-19 disease. In this respect, macrolides like azithromycin are already known HDAC-2 agonists, and theophylline has also been reported to activate this deacetylase.

Bromodomain inhibitors could also be deployed to interrupt another interaction identified in the Nature paper, between SARS-CoV-2 transmembrane envelope protein (E) and BRD2 and/or BRD4. "This potential mechanism of action could be relevant in the context of the secondary immune-related consequences of SARS-CoV-2 infection," says Andy Conery, director of Functional Genomics at Constellation Pharmaceuticals. Those bromodomain inhibitors already in the clinic include Resverlogix’s apabetalone, in phase 3 trials for cancer and phase 1 for pulmonary arterial hypertension; AbbVie's ABBV-744, in phase 1 for cancer; and Constellation Pharmaceuticals' CPI-0610, in phase 2 for cancer.

Overall, though, the lack of selectivity of most small-molecule epigenetic modulators means that side effects are likely to count against their progress in the context of COVID-19. According to Ricky Johnstone of the Peter MacCallum Cancer Centre and University of Melbourne, Australia, "the notion of selectively targeting HDAC-2 as a COVID-19 therapy would be very difficult to prosecute at the moment."

\section{Charlotte Harrison \\ Canterbury, UK}

Published online: 15 May 2020

https://doi.org/10.1038/d41587-020-00013-z 